\title{
PERCUTANEOUS RENAL SURGERY - NEW MODEL FOR LEARNING AND TRAINING
}

\author{
PEDRO P. DE SÁ EARP \\ Section of Urology, Petrópolis School of Medicine, Petrópolis, Rio de Janeiro, Brazil
}

\begin{abstract}
Introduction: Aiming at promoting and facilitating learning and training in percutaneous renal surgery, we have created an easy to assemble, reproducible and cheap laboratory model. The model was built using pig kidney, foam layer, plastic catheter, linen or cotton holding sutures, and wide scotch tape.

Surgical Technique: The kidney with catheterized ureter is fixed and involved in a foam layer. It stays hidden, and is visible only through radioscopy. This model is positioned and fixed to a radiological table in such a way that it simulates the patient's lumbar region. After that, contrast medium is injected through the ureter, and the urinary system is examined through radioscopy. All percutaneous maneuvers can be accomplished, from the puncture, tract dilation, insertion of Amplatz sheath, and introduction of nephroscope, allowing lithotripsy and endopielotomy, as well as other types of percutaneous surgeries.

Comments: The great advantage of this model is its easy construction, by using very cheap and widely available material. Foam can be several times washed and reused. After treatment, the model can be immediately open, and a critical analysis can be made, being then possible to verify if the place of renal puncture was well chosen, if dilation was correctly accomplished, and if the collecting system has been preserved. Therefore, this model can represent a great advance for the learning and training in percutaneous surgery.
\end{abstract}

Key words: kidney; endourology; nephrostomy, percutaneous; models, educational Int Braz J Urol. 2003; 29: 151-4

\section{INTRODUCTION}

The model created for learning and training in percutaneous renal surgery in laboratory allows the accomplishment of renal puncture, tract dilation, Amplatz sheath introduction, nephroscopy, nephrolitotripsy, and other endourological maneuvers. The model can be simply and cheaply built, without the need of sophisticated material, being accessible to any resident or urologist in need of acquiring more experience with this technique.

\section{MODEL AND SURGICAL TECHNIQUE}

We use kidneys from large pigs, whose sizes are most similar to human kidneys. During kidney extraction, we recommend that the maximum of perirenal fat be preserved. This will help the kidney fixation to the foam through perirenal points, without harming them too much. We leave the ureter as long, in order to be catheterized.

We make a foam layer of $3 \mathrm{~cm}$ thick, $60 \mathrm{~cm}$ wide and $50 \mathrm{~cm}$ long. We centralize the kidney in the 
middle of the foam, angled 30 degrees in relation to frontal plane, thus simulating renal position in human beings.

We fix the renal poles with sutures that go through the fat, the renal capsule, through the superficial part of parenchyma and through the foam. It is important not to hit the collecting system, nor deform the kidney (or fasten points too much) nor tear the renal tissue with suture thread. To get a better resistance from renal tissue, it is best to keep the kidney in the freezer one day before, and defrost it few hours

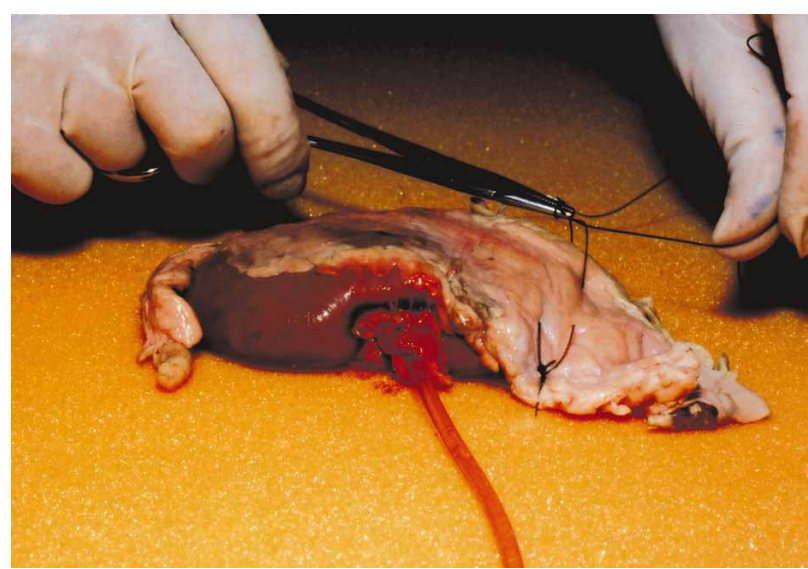

Figure 1 - The kidney is fixed with sutures to the back foam sheet. Note that the ureter is catheterized.

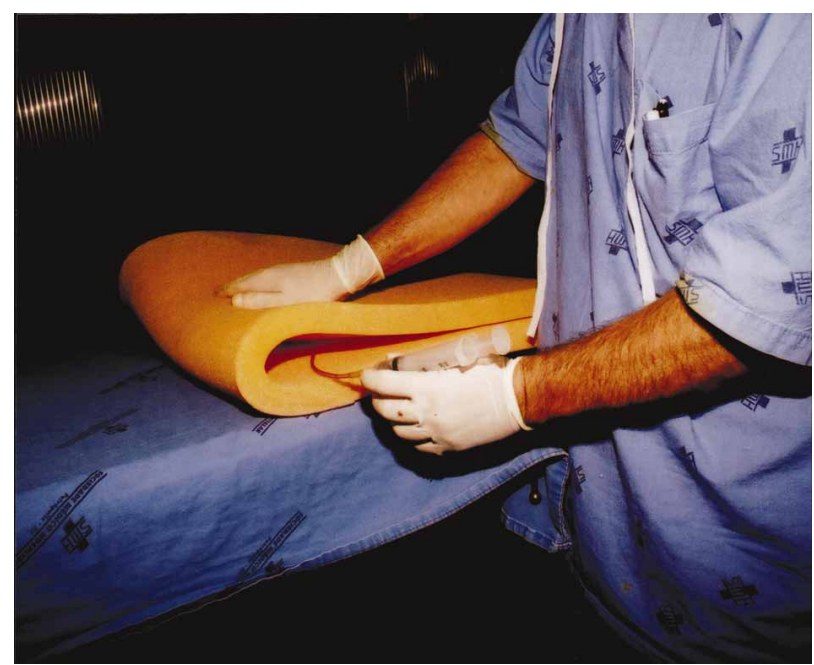

Figure 2 - With the kidney fixed, the foam is folded over it, and then, closed with scotch tape. before the procedure, in such a way that the collecting system is defrosted and the parenchyma is not too soft or friable in the moment of use (Figure-1).

The ureter is catheterized with a plastic catheter $(6 \mathrm{~F})$, which is introduced to the upper ureter, where it is fixed. This catheter will be used for contrast medium injection and must have a longer length than that of the foam, in order to be manipulated from outside the model (Figure-2). Stones can be previously placed inside the kidney through the renal pelvis, which is hermetically sutured afterwards.

We vertically fold the foam, so that the kidney, which is fixed in the back foam sheet, is covered by half of the front sheet. The convex part of the kidney will be in contact with the fold of these sheets

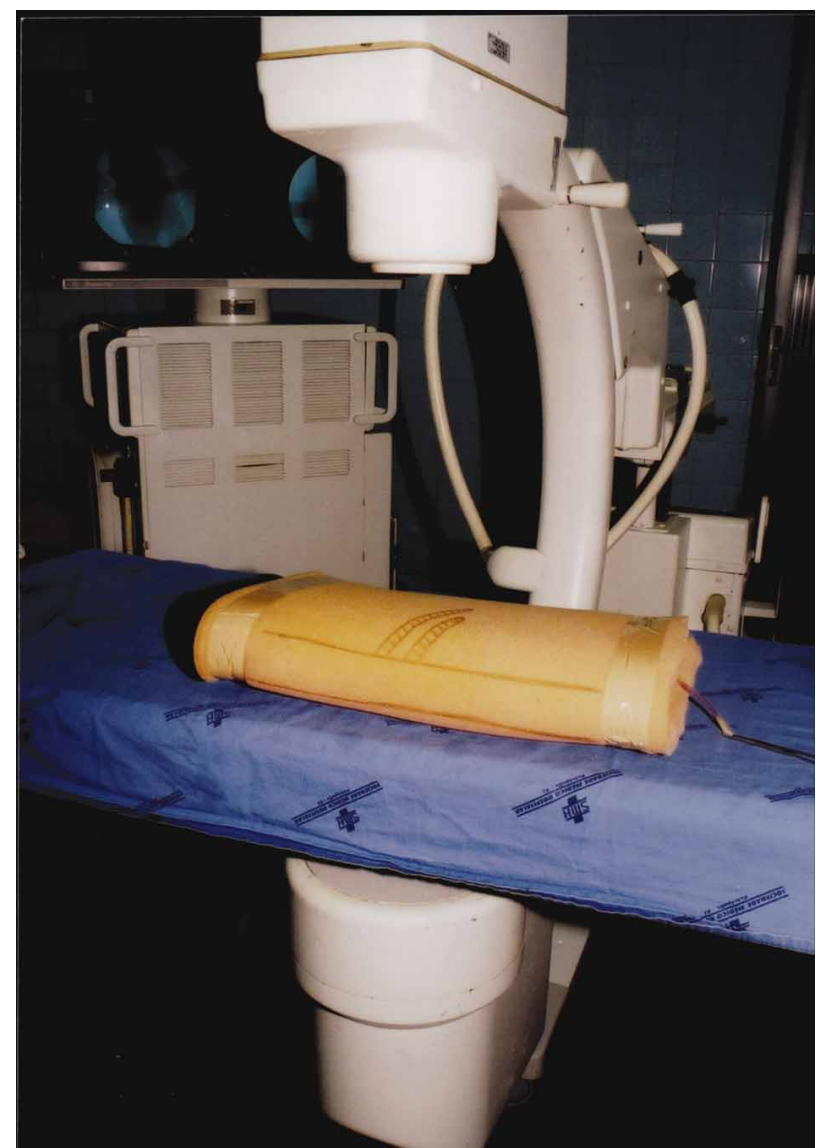

Figure 3 - The model is closed with tape and positioned in the radioscopy table. It is noted the drawing of 11th and 12th ribs, and posterior axillary line. The ureteral catheter is outside the foam. 


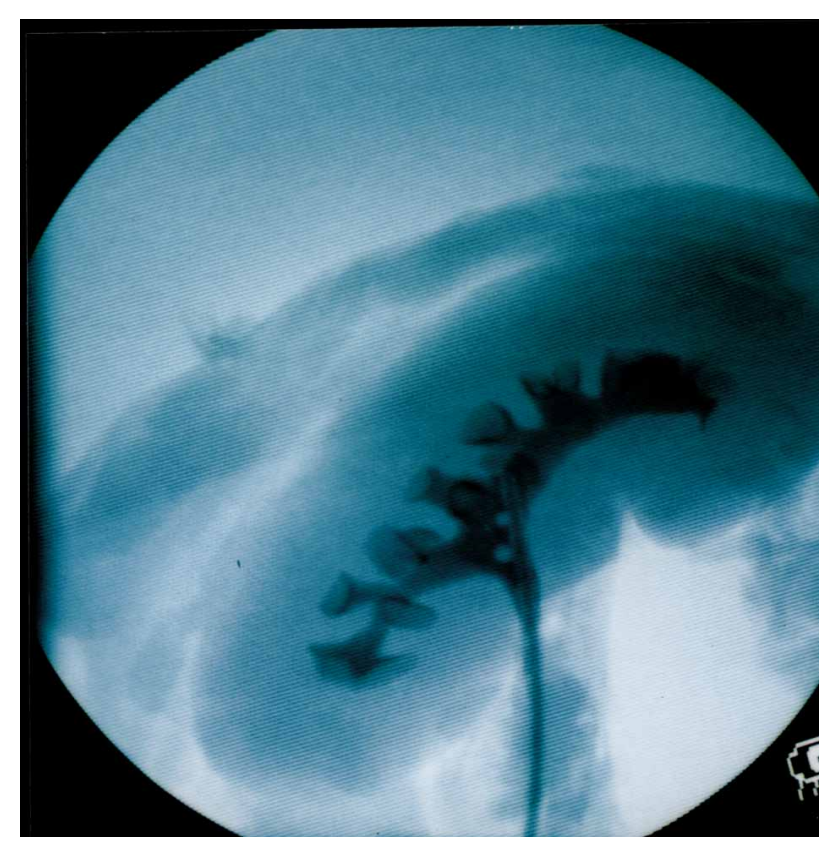

Figure 4 - Retrograde pyelography, showing the renal shadow and the renal collecting system.

(Figure-2). A wide scotch tape is passed twice around the upper and lower part of the folded foam, completely hiding the kidney. Other tapes close the remaining openings. So, a foam envelope is formed around the kidney attached to it. The ureteral catheter is exteriorized in the lower part of this envelope. With a roller ball pen, the ribs and the posterior axillary line are drawn to simulate the lumbar

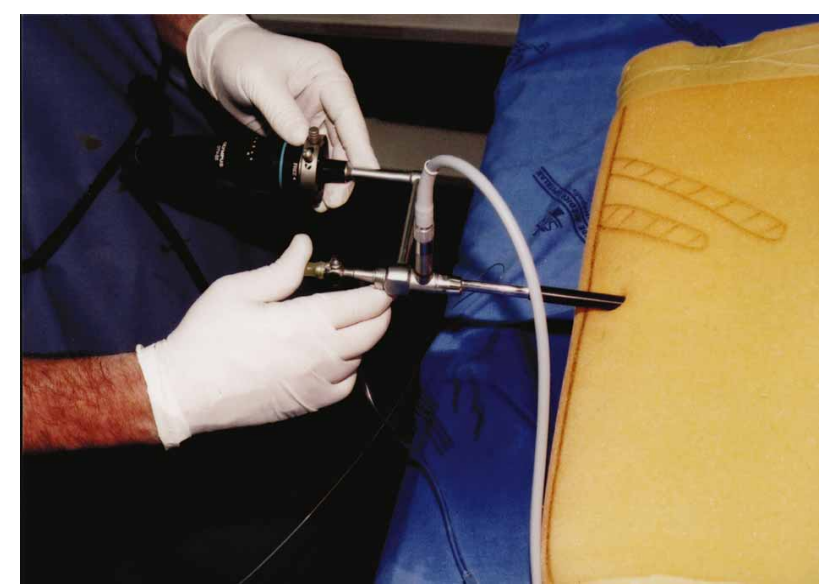

Figure 5 - Amplatz sheath and nephroscope already positioned in the model that is over the radioscopy table. region. The model is fixed to the radiological table, over plastic to avoid wetting the table during training (Figure-3).

Radiological contrast medium is injected to reproduce the complete percutaneous renal surgery (Figure-4). Then we proceed to puncturing, dilation, insertion of Amplatz sheath, and introduction of nephroscope and surgical accessories (Figure-5).

After training, the model can be opened. This allows kidney examination and a critical analysis of the technique employed, speeding up learning (Figure-6).

\section{COMMENTS}

The learning of percutaneous surgery is very difficult, because there is no experimental model that can be easily to acquire and simply to manipulate, as it is the case of black box models used in the training of laparoscopic surgery $(1,2)$.

Although there is some models for learning percutaneous renal surgery that imitate the texture of human tissue, we note that in the present model, the consistency and resistance of the foam offer a good imitation of reality, allowing for training in puncturing and tract dilation (3). The pig kidneys have their anatomy very similar to the one of human beings, exceedingly facilitating, with realism, the intra-renal endoscopic training (4). Before con-

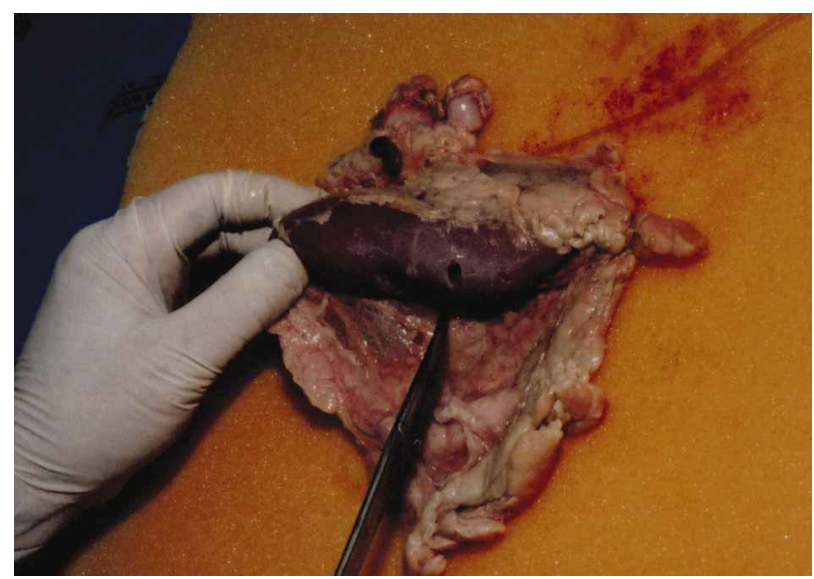

Figure 6 - Kidney examination after training and removal of perirenal fat. The site of intra-renal puncture can be seen. 
struction of the model, stones can be placed inside the renal pelvis, allowing the use of energy sources for lithotripsy.

The great advantage of this model is the easiness of its construction, and especially its cost. Worldwide there are pig kidneys available, foam sheet, suture threads, plastic probes and roller ball pens.

Another advantage is the possibility of opening the model right after its use, in order to make a critical analysis, identify and correct technique mistakes $(5,6)$.

We advice those who do not have any experience with puncturing, to start the training with a model that has the guide-wire previously placed inside the collection system and that exits from the foam. In this way, the training starts by the easiest part, that is dilation, then the introduction of Amplatz sheath and surgical endoscopy. After this training, it is possible to go to the most difficult part, which is the puncture of the calices, by using models without previously placed guide-wires.

\section{REFERENCES}

1. Long JP, Faller GT: Percutaneous cryoablation of the kidney in a porcine model. Cryobiology 1999; 38: 8993.

2. Radley SC, Chapple CR, Martin SW, Boaler D: A training model for transurethral injection therapy for stress incontinence. BJU Int. 1999; 83: 711-3.

3. Travis DG, Tan HL, Webb DR: Single-increment dilatation for percutaneous renal surgery: an experimental study. Br J Urol. 1991; 68: 144-7.

4. Sampaio FJB, Pereira-Sampaio MA, Favorito LA: The pig kidney as an endourological model. Anatomical contribution. J Endourol. 1998; 12: 45-50.

5. Shalhav AL, Dabagia MD, Wagner TT, Koch MO, Lingeman JE: Training postgraduate urologists in laparoscopic surgery: the current challenge. J Urol. 2002; 167: 2135-7.

6. Michel MS, Knoll T, Kurmann KU, Alken P: The URO Mentor: development and evaluation of a new computer-based interactive training system for virtual life-like simulation of diagnostic and therapeutic endourological procedures. BJU Int. 2002; 89: 174-7.

Received: February 28, 2003 Accepted: March 31, 2003

\footnotetext{
Correspondence address:

Dr. Pedro Paulo de Sá Earp

Rua Sorocaba 464 / 403

Rio de Janeiro, RJ, 22271-110, Brazil

Fax: + 5521 2527-9927

E-mail: ppdesaearp@ig.com.br
} 